\title{
PRACTICE BASED LEARNING AND IMPROVEMENT
}

Practice Based Learning and Improvement (PBLI) is a means of evaluating individual and system practice patterns and incorporating the best available evidence to improve patient care. PBLI is recognized as a critical skill for all clinicians by the Accreditation Council for Graduate Medical Education (ACGME), the American Board of Internal Medicine (ABIM), and the American Board of Pediatrics (ABP). As the practice of Hospital Medicine rapidly evolves, hospitalists apply the most up-to-date knowledge to their care of inpatients. Hospitalists use a PBLI approach to lead, coordinate and participate in initiatives to improve hospital processes and clinical care.

\section{KNOWLEDGE}

Hospitalists should be able to:

- Describe systematic methods of analyzing practice experience.

- Explain key concepts of practice based improvement methodology, which include the Plan Do Study Act (PDSA) model.

- Define the role of multidisciplinary teams and team leaders in improving patient care.

- Describe how critical appraisal skills, including study design, statistical methods and clinical relevance apply to PBLI.

- Describe how information technology can be used to identify opportunities to improve patient care.

\section{SKILLS}

Hospitalists should be able to:

- Translate information about a general population into management of subpopulations or individual patients.

- Critically assess individual and system practice patterns and experience to identify areas for improvement and minimize heterogeneity of practice.

- Design practice interventions to improve quality, efficiency, and consistency of patient care using standard PBLI methodology and tools.

- Assess medical information to support self-directed learning.

- Establish and maintain an open dialogue with patients and families regarding care goals and limitations, palliative care, and end of life issues.

- Critically appraise and apply the reports of new medical evidence.

- Use health information systems efficiently to manage and improve care at the individual and system levels.

- Utilize evidence based information resources to inform clinical decisions.

\section{ATTITUDES}

Hospitalists should be able to:

- Advocate for the use of PBLI in clinical practice and in system improvement projects.

- Create an environment conducive to self-evaluation and improvement.

- Advocate for investment in information technology.

- Facilitate and encourage self-directed learning among health care professionals and trainees.

- Promote self improvement and care standardization, utilizing best evidence and practice. 